\title{
Nuclear data for astrophysics: resources, challenges, strategies, and software solutions
}

\author{
Michael S. Smith ${ }^{1,2, a}$, Eric J. Lingerfelt ${ }^{1,2}$, Caroline D. Nesaraja ${ }^{1,2}$, W. Raphael Hix ${ }^{1,2}$, Luke F. Roberts ${ }^{1,2}$, Hiroyuki Koura ${ }^{3}$, \\ George M. Fuller ${ }^{4}$, and David Tytler ${ }^{4}$ \\ 1 Physics Division, Oak Ridge National Laboratory, Oak Ridge, TN 37831-6354, USA \\ 2 Dept of Physics \& Astronomy, University of Tennessee, Knoxville, TN 37996, USA \\ 3 Advanced Science Research Center, Japan Atomic Energy Agency, Tokai, Naka-gun, Ibaraki 319-1195, Japan \\ 4 Dept of Physics, Univ. California San Diego, La Jolla, CA 92093, USA
}

\begin{abstract}
One of the most exciting utilizations of nuclear data is to help unlock the mysteries of the Cosmos - the creation of the chemical elements, the evolution and explosion of stars, and the origin and fate of the Universe. There are now many nuclear data sets, tools, and other resources online to help address these important questions. However, numerous serious challenges make it important to develop strategies now to ensure a sustainable future for this work. A number of strategies are advocated, including: enlisting additional manpower to evaluate the newest data; devising ways to streamline evaluation activities; and improving communication and coordination between existing efforts. Software projects are central to some of these strategies. Examples include: creating a virtual "pipeline" leading from the nuclear laboratory to astrophysics simulations; improving data visualization and management to get the most science out of the existing datasets; and creating a nuclear astrophysics data virtual (online) community. Recent examples will be detailed, including the development of two first-generation software pipelines, the Computational Infrastructure for Nuclear Astrophysics for stellar astrophysics and the bigbangonline suite of codes for cosmology, and the coupling of nuclear data to sensitivity studies with astrophysical simulation codes to guide future research.
\end{abstract}

\section{Background and motivation}

The interdisciplinary field of nuclear astrophysics was established because fundamental knowledge of science at length scales of $10^{-13} \mathrm{~cm}$ is required to address fascinating astrophysical questions such as the origins of the elements that make life possible, the evolution and explosions of stars, and the origin, composition, age, and ultimate fate of the Universe. Indeed, nuclear reaction information (e.g., cross sections, s-factors, reaction rates), nuclear structure information (e.g., masses, decays, resonance properties), and other specialized data sets (e.g., the nuclear equation of state) serve as the empirical foundation of our knowledge of many astrophysical sites and events. The availability of incredible images and data from powerful space- and ground-based observatories (e.g., Hubble, Chandra, Wilkinson, Keck, Subaru, and others), as well as the sophisticated astrophysical simulation codes that run on the fastest supercomputers, have led to a tremendous growth in this field. With new nuclear accelerator laboratories on the horizon (e.g., RIKEN RI Beam Factory, GSI/FAIR, RIA) that promise tremendous amounts of new data, this growth will definitely continue in the future.

This growth is strong motivation to expand efforts to bolster the nuclear foundation of astrophysical studies. Improved nuclear science is needed, for example, to decipher the latest measurements using satellite observatories of longlived radionuclides [e.g., ${ }^{26} \mathrm{Al},{ }^{44} \mathrm{Ti},{ }^{18} \mathrm{~F}$ ] that are synthesized in and dispersed by supernova [1] and/or nova [2] explosions. Improved nuclear data - especially uncertainty and covariance information - is also needed for studies that attempt to quantify what portion of the uncertainties in astrophysical

\footnotetext{
${ }^{a}$ Presenting author, e-mail: smithms@ornl.gov
}

predictions (e.g., for X-ray bursts [3]) is due to uncertainties in the input nuclear physics. New multidimensional astrophysics supercomputer simulations (e.g., for supernovae [4]) require more, and more precise, nuclear data, than ever before. Finally, a combination of astrophysical observations, astrophysical simulations, and nuclear datasets are being synergistically fused in a new generation of studies of the sensitivity of model predictions on input nuclear data (e.g., fig. $1[5,6])$ - and improved nuclear datasets will tremendously aid this work.

There are additional motivations for expanding nuclear data work targeted towards astrophysics. First, this research is an inexpensive and cost effective way to significantly impact our astrophysics knowledge. The cost, for example, of evaluating a reaction is orders of magnitude less than that of measuring the products of that reaction (e.g., by their radioactive decay or their absorbtion spectra) in space. Also, because of wide public appeal of astrophysics, nuclear astrophysics data projects are an excellent way to recruit young researchers into the field of nuclear science. This last point is particularly crucial because of the impending manpower crisis in the nuclear data field - where more than $85 \%$ of professional evaluators are set to retire within the next 10 years and constant funding has resulted in the drop of 2 persons per year over the last 3 years [7]. Finally, this work brings a significant positive exposure to nuclear science research because of its popularity among the general public.

\section{Resources}

The primary astrophysical needs are thermonuclear reaction rates (processed cross sections), as well as nuclear masses, 

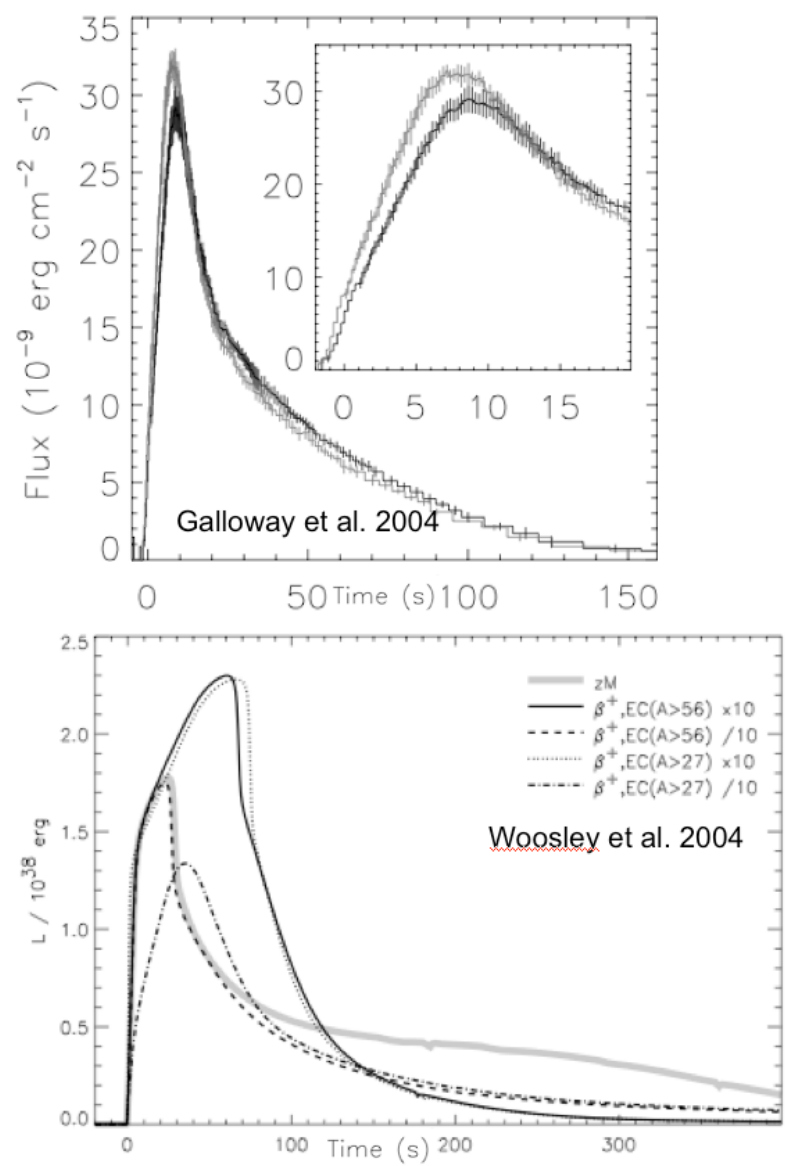

Fig. 1. The variation of X-ray flux in time from X-ray burst RXTE GS1826-24 [5], top, compared to the predictions of theory [6] using different input nuclear data sets, bottom.

levels, decays, and partition functions to calculate forward and inverse reactions and equilibrium populations. Additionally, it is crucial to provide online access to other essential components of astrophysical research, such as observations, simulations, and visualizations of simulation output.

Since the first nuclear astrophysics data website went online in 1995, many more sites have been made, along with $\sim 70$ datasets that are useful for research in nuclear astrophysics. This includes both information specifically for astrophysics (e.g., thermonuclear reaction rates) as well as more general purpose nuclear physics information (e.g., nuclear masses, nuclear level information) needed for astrophysics simulations. There is now some information online in each of the resource categories mentioned above, and they are all hyperlinked at the online gateway site nucastrodata.org (fig. 2).

\section{Challenges}

While it is unrealistic to expect complete, up-to-date, evaluations of all the information needed for astrophysics research, the current situation is far from ideal. One striking example: the last "official, public" update of the most widely used library of thermonuclear reaction rates, REACLIB, was in 1995. This valuable resource, with over 60000 rates, does not

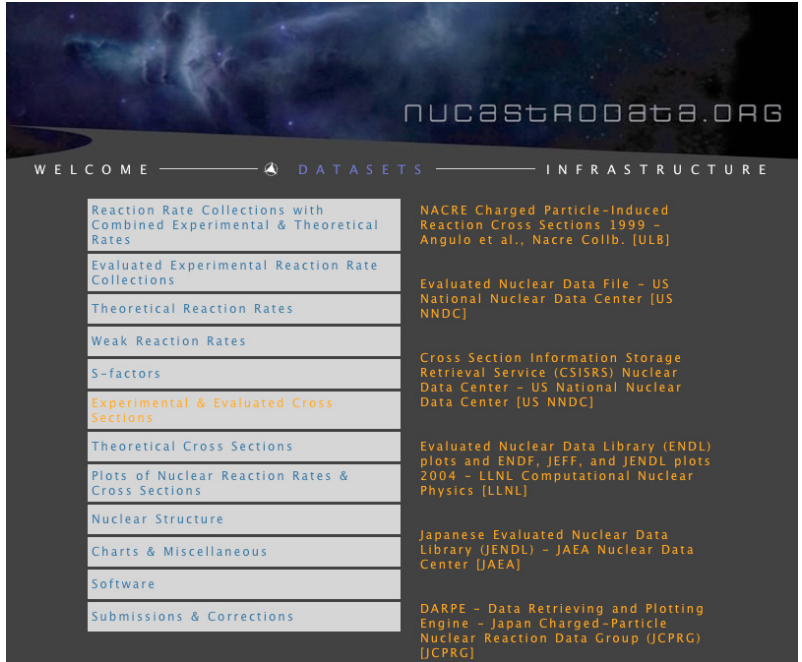

Fig. 2. Nuclear astrophysics datasets hyperlinked at nucastrodata.org.

include any experimental information from the last 12 years. Furthermore, this is not the only example of a long time lag between lab measurements and incorporation into "leading" databases or compilations. As a result, astrophysics modelers must now often rely on nuclear information that is years out of date. Not only does this make their model predictions outdated and therefore questionable, it also severely limits the scientific return on investment for many experimental and theoretical nuclear astrophysics projects.

How did this situation arise? While the number of online datasets has recently increased, the manpower and funding to update and expand these valuable collections have steadily decreased. Also, while the data needs of the community were well met 30 years ago by one single effort led by Willy Fowler, current data needs are so extensive that they cannot be met by one, or even a few, groups. Current astrophysics data efforts are overwhelmingly independent, voluntary, underand haphazardly-funded, and driven by specific short-term research projects. As a result, they understandably lack continuity, completeness, and a coordinated vision for the future. Another issue is competition: with a lack of regularly-updated "public" datasets, researchers have focussed on improving their own datasets, and then are often reluctant to share these with others before they recover their investment. Furthermore, errors in datasets are not regularly communicated to fellow researchers, and some research efforts are duplicated be cause of a lack of communication and coordination. Visualization is another important issue: the lack of fast, easily-accessible visualization tools for nuclear datasets and simulation predictions slows research progress and frustrates practitioners. Finally, a lack of public datasets and standards makes intercomparison of astrophysical simulations extremely difficult.

Future developments will only exacerbate these issues. New unstable nuclear beam facilities coming online will explore more of the nuclear landscape and generate unprecedented amounts of data; more precise data from astrophysical observatories demand higher precision predictions from astrophysical simulations; and the simulations themselves require more, and more precise, data as input. 


\section{Strategies}

What is the best course of action to address these issues? Because the different researchers involved [data providers, data evaluators, and data end-users] need different nuclear data resources and features for their work, no single data archive, collection, or effort will satisfy them all. Furthermore, the immense amount of data that needs to be compiled, evaluated, processed, and distributed cannot all be handled in any effort with realistic funding levels. Careful strategies - including but going beyond increasing manpower - are therefore required now to ensure that the most important projects are carried out in the most efficient and cost-effective manner possible. Devising such strategies will ensure that this work has a sustainable future.

We first list a number of actions to address each of the challenges mentioned in the last section, then provide additional details in the subsections below. Consider the three issues of outdated and incomplete nuclear data sets, of unprecedented amounts of new nuclear data in the near future, and of more nuclear data needed by simulations. These can all be alleviated by significantly increasing the pace at which evaluations are done. More manpower for evaluations will certainly help, but in an era of constrained budgets, reliance on this alone is not prudent. An increased evaluation pace can also be achieved by speeding up individual evaluation projects. Furthermore, the pace can be effectively increased by prioritizing projects, coordinating efforts, improving communication between researchers to minimize duplication of efforts, and shifting attitudes in the research community. A number of these actions can be significantly aided by software efforts.

Regarding the issues of the different needs of the different communities (nuclear, astrophysics, data) and the lack of a coordinated vision, these can be addressed by improving coordination and communication, as well as by shifting the attitudes of researchers. Finally, the issues of proprietary data sets, of dataset errors not disseminated to the community, of the difficultly in comparing simulations, and of inadequate data/simulation visualization can all be addressed with software solutions, as described in detail below.

\subsection{Evaluation manpower}

Evaluation manpower can be boosted both by obtaining funding for new hires and by diverting some existing manpower towards nuclear astrophysics data work. Candidates can be drawn from any of the nuclear data, nuclear astrophysics, or general nuclear physics communities. To make the positions attractive to young researchers, they should involve both basic research and evaluation work. Also, it is crucial to transfer knowledge from experienced evaluators to those new to the activity, so a mandatory training period will be necessary. Previous proposed strategies, such as the Mentoring in Nuclear Information Technology (MINIT) initiative [8], have recommended that each new recruit be mentored by an experienced evaluator.

Additional recruiting "incentives" include: giving ample credit to evaluators (whose work is often anonymously incorporated within large libraries); encouraging evaluations that are optimized for astrophysics (discussed below); developing evaluation "standards"; being somewhat flexible on evaluation formats; and rapidly and creatively disseminating evaluations once completed. One mechanism to encourage existing manpower to shift towards this work by establishing an "Editorial Board" and publishing results in an online journal has been recently discussed [9].

Another approach to increase manpower is to shift attitudes in the research community. Instead of perpetuating the separation of data evaluation work from measurements, effort should be made to convince experimentalists to evaluate the reactions that they measure. This can be considered part of their "community service", just as reviewing articles for publication is. Furthermore, since some truncated evaluation work is usually done during the planning stages of a measurement, it would not be too much effort to expand on this to complete an evaluation after a measurement is complete. While this approach is susceptible to bias, it also pairs researchers to reactions they are passionate and knowledgeable about.

\subsection{Streamlining evaluations}

Traditional nuclear data evaluations, for entry into the ENSDF (nuclear structure) and ENDF (nuclear reaction) databases, involve complete assessments of all aspects of a nucleus and/or its interactions. For example, structure evaluations are often done in mass chains where all nuclei with the same mass are simultaneously assessed, and all levels are assessed; reaction evaluations, meanwhile, typically involve examining all reaction channels and partial, differential, and total cross sections. While these approaches are the most desirable, much of the resulting information cannot be used in astrophysics calculations. For example, only the total cross section of a reaction is needed to directly determine the thermonuclear rate that is used in astrophysical simulations, and properties of levels less than $\sim 2 \mathrm{MeV}$ above the particle threshold are the most important to indirectly determine a rate. The pace of evaluations can, in most cases, be significantly increased by focussing on just the nuclear informati on needed for input into astrophysical simulations - as long as consistency (e.g., with unassessed reaction channels and/or levels ) is maintained.

Another approach to streamlining evaluations involves automating some of the time-consuming and repetitive tasks that must be routinely completed. These include: uploading, storing, plotting, and renormalizing cross sections and s-factors; extrapolating experimental cross sections with theoretical calculations; calculating thermonuclear reaction rates from cross sections and s-factors; parameterizing reaction rates or generating rate values on a temperature grid; plotting and modifying reaction rates; inserting rates into new or existing rate collections (libraries); and creating, storing, modifying, documenting, and merging libraries. A major recent milestone in streamlining evaluation work was the creation of the Computational Infrastructure for Nuclear Astrophysics [10], the first software "pipeline" from laboratory results to astrophysical simulations. This software suite, online at nucastrodata.org, includes capabilities for file manipulation/management, data processing, simulation execution, data and simulation visualizations, and the sharing and commenting on data and 


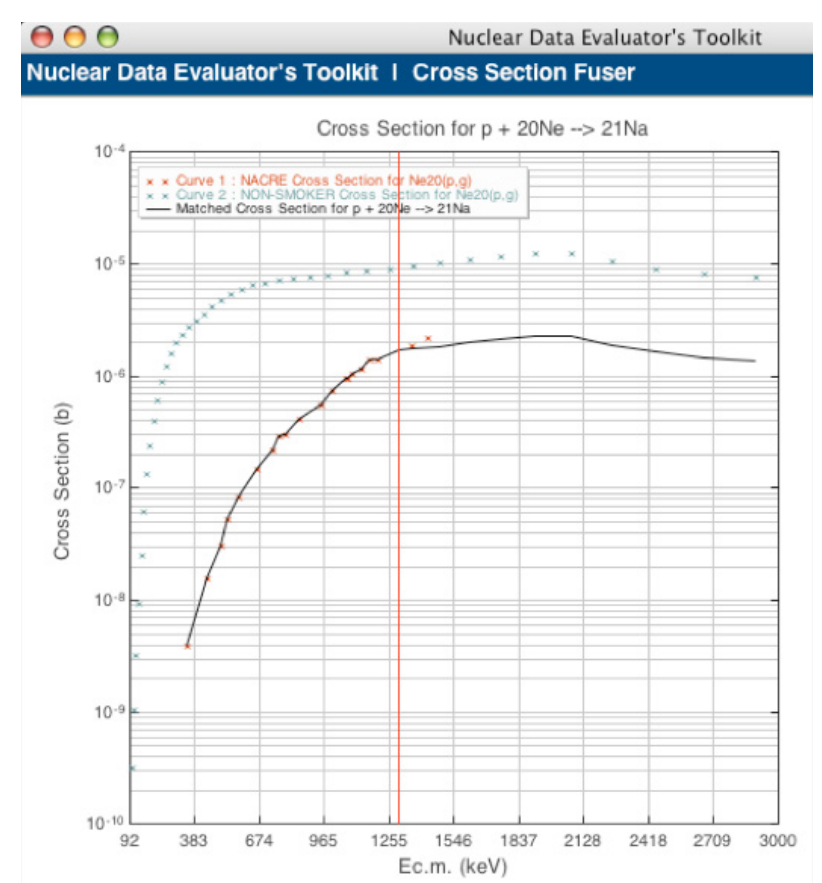

Fig. 3. Extrapolating an experimental cross section with a theoretical one using the Data Evaluation Toolkit in the Computational Infrastructure for Nuclear Astrophysics [10].

simulations. Scientists from 53 institutions in 18 countries are currently using the suite to quickly (within minutes) complete all of the tasks listed above, as well as many others - such as running and visualizing astrophysical simulations, sharing their work with the research community, and commenting on their own work and the work of others. The suite features a Data Evaluation Toolkit to process nuclear data (e.g., fig. 3), and more features are being added on the recommendation of the users. While this suite focusses on stellar evolution and explosions, a related suite at bigbangonline.org [11] enables similar work streamlining for early universe studies.

\subsection{Prioritization}

With limited manpower, prioritization of evaluation projects is extremely important. The primary criteria should be the importance to astrophysics, and studies of the sensitivity of astrophysical simulations on their input nuclear data are invaluable here. The level of sophistication of such studies has significantly increased in the last decade. Some examples: $\mathrm{X}$-ray burst simulations (with coupled hydrodynamics and nuclear networks) with different ${ }^{15} \mathrm{O}(\alpha, \gamma){ }^{19} \mathrm{Ne}$ reaction rates [12] and examining overall reaction flows [13]; core collapse supernova simulations with different ${ }^{132} \mathrm{Sn}(\mathrm{n}, \gamma){ }^{133} \mathrm{Sn}$ rates [14]; Type II supernova simulations with different weak reaction rates [15]; and multi-zone post-processing nova simulations with different ${ }^{17} \mathrm{~F}(\mathrm{p}, \gamma){ }^{18} \mathrm{Ne}[16]$ and ${ }^{18} \mathrm{~F}(\mathrm{p}, \alpha){ }^{15} \mathrm{O}$ [17] reaction rates. For the future, it is imperative to vigorously pursue sensitivity studies with even mo re sophisticated models and, especially, more complete nuclear data sets.

Because of the utility of these studies for prioritizing evaluation projects, measurements. and other theoretical

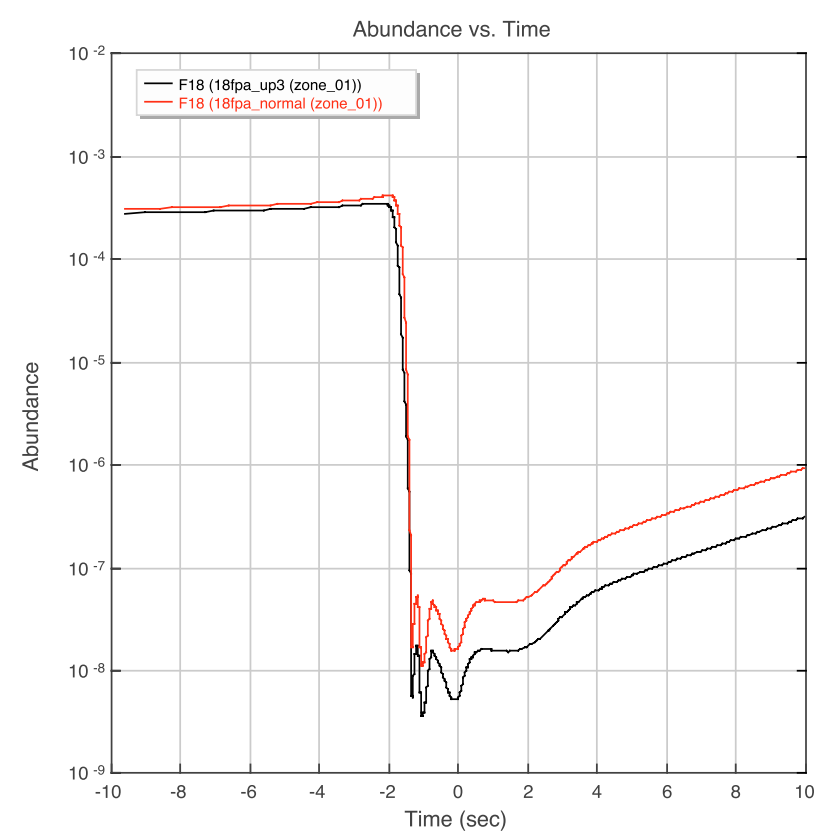

Fig. 4. Comparing predictions of nucleosynthesis of ${ }^{18} \mathrm{~F}$ in novae as a function of time, based on input reaction rate libraries with values of the ${ }^{18} \mathrm{~F}(\mathrm{p}, \alpha){ }^{15} \mathrm{O}$ reaction rate differing by a factor of 3 , using the Computational Infrastructure for Nuclear Astrophysics [10].

calculations, it is imperative to design software systems that enable anyone to run and visualize these calculations. The Computational Infrastructure for Nuclear Astrophysics features explosive element burning ("reaction network" [18]) calculations using a post-processing approach [16] wherein the temperature and density evolution in time are pre-determined from separate, lengthy simulations where hydrodynamics and nuclear effects are fully coupled [19]. The suite facilitates sensitivity studies by enabling quick comparisons of the results of explosive nucleosynthesis simulations that utilize different, custom input nuclear data sets created by the User (fig. 4). This translates nuclear reaction rate changes into changes in nuclear abundances, a quantity of observational interest.

In traditional sensitivity studies, one or a few reactions are varied at a time. For systems such as stellar explosions, - where hundreds or thousands of reactions are used as input, a quantifiable strategy is needed to select a very small subset of all possible reactions to vary. Alternatively, a Monte Carlo approach can be used, where thousands of nucleosynthesis trials are run in which every input reaction is varied randomly and independently. The variations are chosen in such a way that, over all of the trials, there is a lognormal distribution of variations with a width given by the inherent uncertainty of the rate. This approach enables uncertainties to be assigned to the model predictions (e.g., final abundances, light output), and also easily determines the correlation between the input nuclear data input and the model output. Such studies have been carried out for the Big Bang for years (e.g., [20]), and are now accessible online to anyone at the bigbangonline suite (fig. 5). Monte Carlo studies are also now being pursued for nova explosions [21] and X-ray bursts [3] (fig. 6).

Simulations, however, are not the only criteria to determine the priority of evaluation projects. Other important 


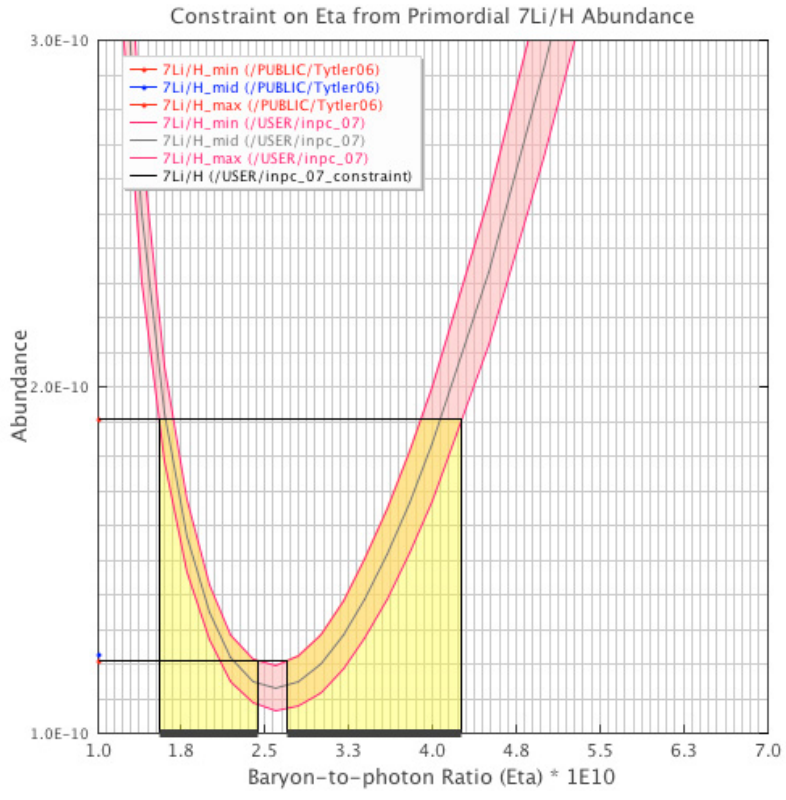

Fig. 5. Constraint on baryon-to-photon ratio by comparing observed primordial ${ }^{7} \mathrm{Li} / \mathrm{H}$ abundance with prediction of standard big bang theory, using the Monte Carlo simulation in bigbangonline.org.

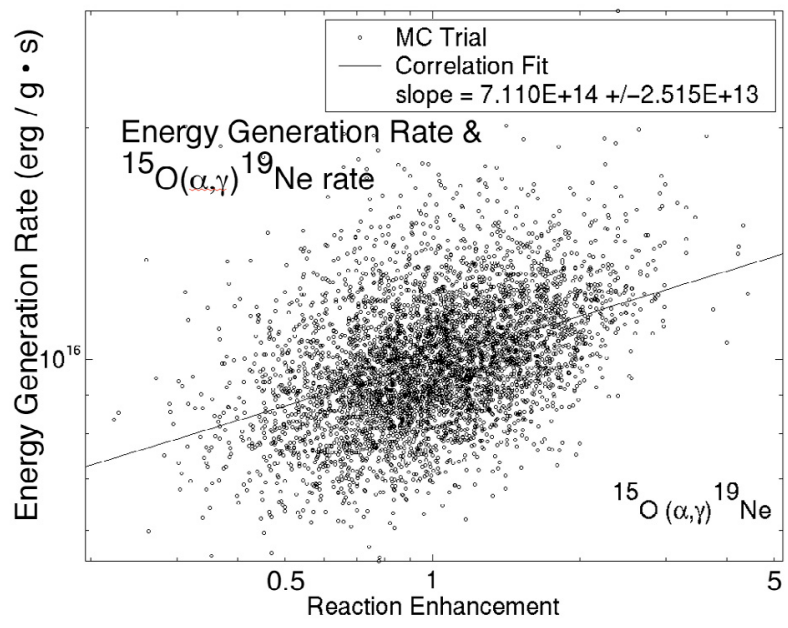

Fig. 6. Positive correlation between X-ray burst energy generation with the ${ }^{15} \mathrm{O}(\alpha, \gamma){ }^{19} \mathrm{Ne}$ reaction rate using a Monte Carlo nucleosynthesis approach [3].

criteria include the activity of experimentalists, the quality of the existing datasets, the number of astrophysical modelers who require the information, the manpower required for an evaluation project, and the availability of scientists interested in performing the evaluations. Some of these prioritization criteria are being addressed by an ad-hoc Working Group discussed below.

\subsection{Enhanced visualization tools}

The overwhelming majority of the online nuclear data resources for nuclear astrophysics have no associated visualization tools. While some have limited to moderate capabilities

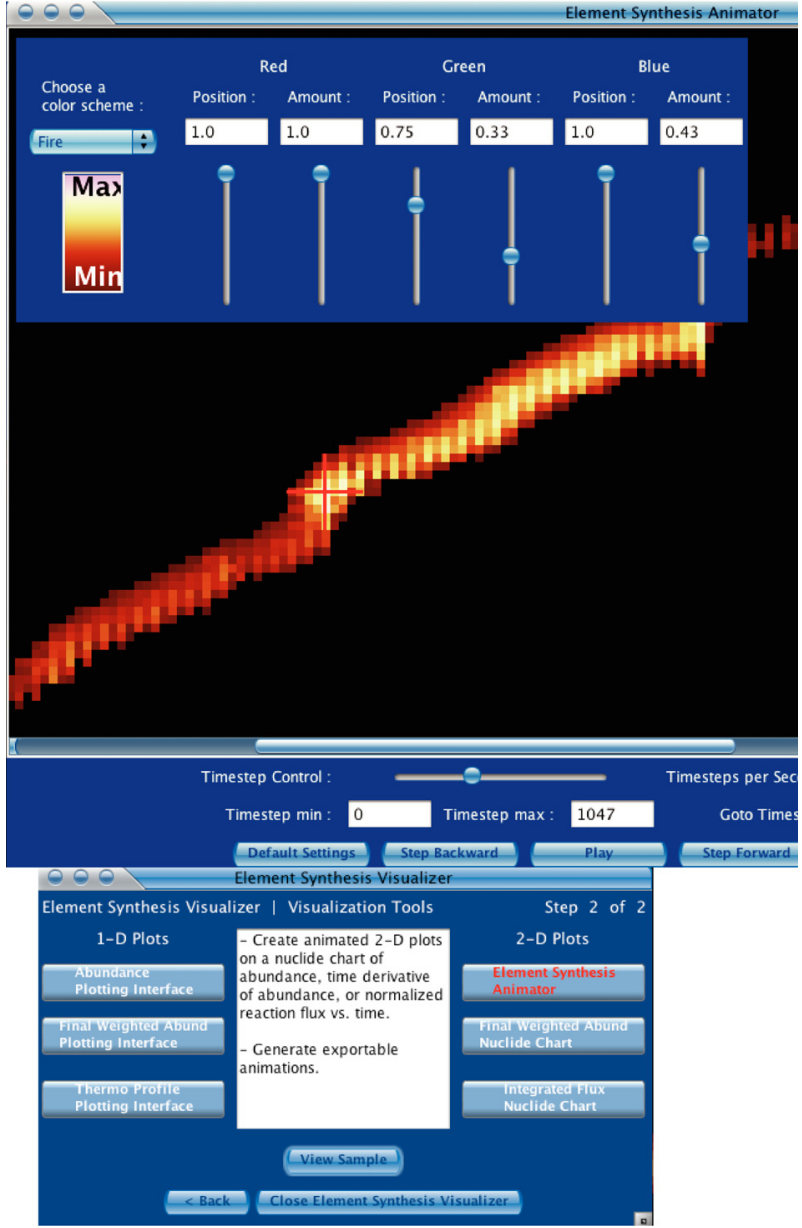

Fig. 7. Screenshot of animated nuclide chart showing evolution of abundances in the r-process in supernovae as a function of time in the Computational Infrastructure for Nuclear Astrophysics [10]. Also shown are windows to customize the color mapping of the abundances (top inset) and to choose different plot types (bottom inset).

and a few are excellent, much effort is needed to make powerful, easy-to-use visualization tools widely available to nuclear science researchers. Within the Computational Infrastructure for Nuclear Astrophysics [10], there are capabilities for 1D plots of nuclear information (cross sections (fig. 3), s-factors, mass excesses), processed information (reaction rates), and astrophysical simulation results (abundance predictions as a function of time (fig. 4)). There are also customizable 2D plots of nuclear information (masses, separation energies) and astrophysical simulation results (abundances (fig. 7), abundance time derivatives, reaction flows), the latter of which are animated to show the evolution of predictions in time. These unique capabilities, which will be commonplace in the future, greatly speed up many portions of the workflow in nuclear astrophysics research.

\subsection{Coordination and communication}

Improving communication between existing nuclear astrophysics data efforts is another way to increase the pace at which evaluations are completed - and is especially important 
when evaluation manpower is limited. Enhanced communication is crucial to: publicize new evaluation techniques and codes; report on work in progress; transfer knowledge to a new generation of evaluators; avoid unnecessary duplication; arrange collaborative efforts; broadcast the latest experimental results; and disclose errors in databases. A natural way to enhance communication is to coordinate efforts. Coordination also involves discussions of project priorities and of matching projects to the capabilities of different groups. While rigid coordination can be effective in efforts with one funding source (e.g., a large, multi-institution proposal such as NACRE [22]), it is not possible for distributed, independently-funded efforts.

Before 1995, nuclear astrophysics data efforts in the US were quite independent. With the formation of the Astrophysics Task Force of the US Nuclear Data Program (USNDP), coordination and communication was enhanced among USNDP members. However, there is still a need to fold in other efforts in the US and abroad. One technical solution was the launching of an online forum (nucastrodata.org/forum) where researchers can share information on projects, techniques, software, and other details that may not normally make it into the published literature. This is the first step towards the creation of a virtual online nuclear astrophysics data community. Another technical solution is found within the Computational Infrastructure for Nuclear Astrophysics: researchers can add, browse, and reply to comments on datasets using an email-type interface. This feature enables scientists to reach consensus on some issues (e.g., which particular rate is best for a given reaction) as well as to further build an on line community. A very different approach was initiated in 2006 with the formation of an ad-hoc, international Working Group to examine the worldwide status of nuclear data for nuclear astrophysics studies and recommend a path forward. Working Group meetings will continue in 2007, with members that currently include researchers from institutions in the US and Europe. Issues being discussed include updating popular reaction rate databases (e.g., REACLIB), dissemination strategies, and methods to enlist and retain evaluators. The online forum mentioned above also supports the activities of the Working Group.

\section{Summary}

Numerous reasons to expand existing nuclear information science efforts to provide data for astrophysics studies are presented. These include new nuclear facilities on the horizon promise volumes of new data, and new sophisticated astrophysics simulations and nuclear models require more, and more up to date, data. Nuclear astrophysics data projects are an inexpensive and cost effective way to significantly impact astrophysics research, while simultaneously serving as an excellent way to recruit young scientists into nuclear science. We detailed a number of strategies to increase manpower, streamline activities, and improve communication and collaboration. Software solutions are now helping address many of these issues and will become even more important in the future. Two prime examples are given: first-generation software "pipelines" from lab results to astrophysical simulations have now been developed: the Computational Infrastructure for Nuclear Astrophysics for stellar astrophysics and the bigbangonline suite of codes for cosmology. These, along with the coupling of nuclear data to sensitivity studies with astrophysical simulation codes, illustrate the powerful way in which nuclear data impacts and guides future research in astrophysics.

ORNL is managed by UT-Battelle, LLC, for the US Department of Energy under contract DE-AC05-00OR22725.

\section{References}

1. R. Diehl, F.X. Timmes, Publ. Astron. Proc. Pacific 110, 637 (1998).

2. M.J. Harris et al., Astrophys. J. 522, 424 (1999).

3. L.F. Roberts, W.R. Hix, M.S. Smith, J.L. Fisker, in Proc. Int. Symp. Nuclear Astrophysics - Nuclei in the Cosmos IX, Proceedings of Science, June 2006, http://pos.sissa.it//archive/ conferences/028/202/NIC-IX_202.pdf.

4. C. Travaglio, K. Kifonidis, E. Muller, in Carnegie Observatories Astrophysics Series, Vol. 4: Origin and Evolution of the Elements, edited by A. McWilliam, M. Rauch (2003), Pasadena: Carnegie Observatories, http://www.ociw.edu/ociw/ symposia/series/symposium4/proceedings.html.

5. D.K. Galloway et al., Astrophys. J. 601, 466 (2004).

6. S.E. Woosley et al., Astrophys. J. Suppl. 151, 75 (2004).

7. P. Oblovinsky et al., in US Nuclear Data Program 2007 Work Plan (2007); http://www.nndc.bnl.gov/usndp/docs/usndpfy06_ fy08planfinal.pdf

8. M.S. Smith, R.A. Meyer, IAEA International Nuclear Data Committee Technical Meeting Summary Report INDC(NDS)456 (2004) 145; http://www-nds.iaea.org/reports-new/indcreports/indc-nds/indc-nds-0456.pdf

9. H. Schatz (2004) (unpublished).

10. M.S. Smith et al., in Proc. Int. Symp. Nuclear Astrophysics - Nuclei in the Cosmos IX, Proceedings of Science, June 2006, http://pos.sissa.it//archive/conferences/028/180/NICIX_180.pdf.

11. bigbangonline.org

12. J.L. Fisker, J. Gorres, M. Wiescher, B. Davids, Astrophys. J. 650, 332 (2006).

13. J.L. Fisker, H. Schatz, F.-K. Thielemann, astro-ph/0703311.

14. J. Buen et al. (2007) (in preparation).

15. W.R. Hix et al., Phys. Rev. Lett. 91, 201102 (2003).

16. S. Parete-Koon et al., Astrophys. J. 598, 1239 (2003).

17. K. Chae et al., Phys. Rev. C 74, 012801R (2006).

18. W.R. Hix, F.-K. Thielemann, J. Com. Appl. Math. 109, 321 (1999).

19. M. Politano et al., Astrophys. J. 448, 807 (1995).

20. M.S. Smith, L.H. Kawano, R.A. Malaney, Astrophys. J. Suppl. 85, 219 (1993).

21. M.S. Smith, W.R. Hix, L.F. Roberts, S. Starrfield, D.L. Smith (2007) (in preparation).

22. C. Angulo et al., Nucl. Phys. A 656, 3 (1999) 